\title{
Pruebas Iniciales para Construir Tarjetas Electrónicas Biodegradables.
}

Uscanga Villalba, Jorge Miguel Electro Systems and Technological Solutions S.A. de C.V., México juscanga@gmail.com

Hernández Xolo, Ramón Electro Systems and Technological Solutions S.A. de C.V., México herxolo88@gmail.com
Resumen - El aumento de residuos sólidos urbanos puede explicarse como resultado de múltiples factores, entre los más importantes el crecimiento urbano, el desarrollo industrial, las modificaciones tecnológicas y el cambio en los patrones de consumo de la población entre otras. Esta relación que se observa en otras regiones del mundo, se traduce en que a mayores niveles de consumo mayor volumen de residuos. En el caso mexicano, entre 2003 y 2015 , el producto interno bruto y la generación de residuos crecieron prácticamente a la misma tasa. No obstante, en algunos casos la contribución a la producción total nacional de RSU de algunas entidades se desvía notablemente de la tendencia general. Los factores importantes a considerarse al seleccionar un material alterno para la elaboración de tarjetas electrónicas es el grado de absorción de humedad, resistencia de aislamiento y constante dieléctrica.

Palabras claves - Residuos; Tarjetas Electronicas; Sustrato Biodegradable;
López Zavaleta, Roxana Electro Systems and Technological Solutions S.A. de C.V., México biologa.roxana.10@gmail.com

Abstract - The increase in urban solid waste can be explained as a result of multiple factors, among the most important urban growth, industrial development, technological changes and changes in consumption patterns of the population among others. This relationship is observed in other regions of the world, which means that at higher levels of consumption, greater volume of waste. In the Mexican case, between 2003 and 2015, the gross domestic product and the generation of waste grew at practically the same rate. However, in some cases the contribution to the total national production of RSU by some entities deviates markedly from the general trend. The important factors to be considered when selecting an alternative material for the elaboration of electronic cards is the degree of humidity absorption, insulation resistance and dielectric constant.

Keywords - Waste; Electronic Cards; Biodegradable Substrate; 


\section{INTRODUCCIÓN}

La utilización y producción de equipos electrónicos aumenta de manera acelerada a nivel mundial en todos los ámbitos en los que se desenvuelve el ser humano. Los beneficios del uso de dichos equipos contribuyen al desarrollo tecnológico e industrial además de brindar comodidad en su vida cotidiana (Belloch, 2000). Sin embargo, así como su uso ha ido en aumento, de igual manera se incrementan los residuos electrónicos ocasionados por la sustitución de equipos debido a la comercialización de nuevas versiones, renovación o actualización de piezas y desecho de los mismos al término de su vida útil.

La generación de basura electrónica constituye un grave problema al planeta y el ambiente, por citar un ejemplo: cada monitor de computadora o pantalla de televisor contiene entre 2 y 8 libras de plomo (Martínez Rivera, 2008), los cuales, al ser desechados, tendrán como fin llegar a los mantos freáticos, y posteriormente, al agua, la cual es utilizada en algunas zonas para consumo humano generando enfermedades a largo plazo.
De manera general, los residuos eléctricos y electrónicos están conformados por tarjetas de circuitos(Gupta, Gupta, \& Tiwari, 2015), las cuales están elaboradas comúnmente sobre un laminado denominado sustrato; este puede estar conformado de diferentes materiales poliméricos reforzados con otros materiales formando lo que se conoce como un composite(Mexicana, Carrillo, Pr, Toluca, \& Licenciatura, 2009). Dichos materiales generalmente provienen de fuentes no renovables (petróleo), causando gran impacto al medio ambiente al no poseer un grado de biodegradabilidad. De este modo, al término de su vida útil, el sustrato utilizado para la elaboración de tarjetas electrónicas queda prácticamente inservible (resina epoxi, fibra de vidrio y baquelita), el cual resiste altas temperaturas y es insoluble en la mayoría de los solventes, por lo que su reciclaje es prácticamente imposible ya que estos no se deforman con el calor, y esto impide fundirlos con facilidad y crear nuevos productos.

De ahí surge la importancia de encontrar sustratos alternativos, con propiedades biodegradables a 
Pruebas Iniciales para Construir Tarjetas Electrónicas Biodegradables.

fin de reducir el impacto ambiental, permitiendo reutilizar una tarjeta electrónica en su totalidad o disminuyendo la acumulación de basura electrónica generada en los rellenos sanitarios si se construyen con materiales ecológicos.

\section{PROBLEMÁTICA EN LA GENE- RACIÓN DE RESIDUOS SÓLIDOS URBANOS}

Entre los factores de riesgo que deben considerarse se encuentran: Los sitios de confinamiento de RSU son importantes generadores de biogases, algunos de los cuales también son gases de efecto invernadero (GEI). Los que se producen en mayor proporción son el metano $(\mathrm{CH} 4)$ y el bióxido de carbono (CO2), mientras que los que se producen en cantidades muy pequeñas son el nitrógeno (N2), sulfuro de hidrógeno (H2S), hidrógeno $(\mathrm{H} 2)$ y oxígeno $(\mathrm{O} 2)$, y en cantidades traza, monóxido de carbono (CO), amoniaco (NH3), hidrocarburos aromáticos y cíclicos y un grupo de gases conocidos como compuestos orgánicos volátiles (COV). Todos ellos generan problemas ambientales de diversa índole, que van desde olores desagradables hasta la contribución al aumento de la temperatura global. Además, varios de esos gases (por ejemplo, el $\mathrm{NH} 4$, el $\mathrm{CO}$ y el CO2) tienen un efecto directo nocivo sobre la salud humana(Martínez Rivera, 2008).

Las sustancias agotadoras de ozono (SAO) son compuestos que afectan la capa de ozono y contribuyen a su destrucción; entre ellos se encuentran los clorofluorocarbonos (CFC), hidroclorofluorocarbonos (HCFC), hidrofluorocarbonos (HFC) y los halones, por mencionar los más importantes. Los SAO se utilizan para fabricar gases refrigerantes utilizados en los refrigeradores y aires acondicionados, por ejemplo, pero también se utilizan en espumantes y aerosoles. Cuando los envases vacíos o los aparatos electrodomésticos que los contienen son descartados de manera inadecuada, los SAO que contienen se liberan a la atmósfera y afectan a la capa de ozono(Molina \& Rowland, 1974).

Muchos RSU generan líquidos durante su proceso de descomposición, los cuales se conocen con el nombre de lixiviados. Su composi- 
ción puede ser muy diversa, y está directamente relacionada con la naturaleza de los residuos de los que provienen; de esta manera, los desechos orgánicos producirán lixiviados de características muy diferentes a aquellos que se generan por la fuga de los materiales con que se elaboran las pilas, por ejemplo. Ya sea que se trate de lixiviados de origen orgánico o no, su composición y cantidad suele representar un riesgo de contaminación para el suelo y los cuerpos de agua adyacentes, tanto superficiales como subterráneos, y pueden provocar problemas de toxicidad, eutrofización y acidificación, por lo que evitar su flujo superficial e infiltración es de suma importancia(Allen, 2001; Corral Torres, Barrientos Becerra, Hernández Berriel, Gómez Beltrán, \& Macedo Miranda, 2011).

\section{MÉXICO: PRODUCCIÓN DE RSU}

En México, según la cifra más reciente publicada en 2015, la generación de RSU alcanzó 53.1 millones de toneladas, lo que representó un aumento del $61.2 \%$ con respecto a 2003 (10.24 millones de toneladas más generadas en ese período). Si se expresa por habitante, alcanzó 1.2 kilogramos en promedio diariamente en el mismo año.

El aumento en la generación de residuos sólidos urbanos puede explicarse como resultado de múltiples factores, reconociéndose entre los más importantes el crecimiento urbano, el desarrollo industrial, las modificaciones tecnológicas y el cambio en los patrones de consumo de la población entre otras.

En México, como en el caso de muchos otros países, el crecimiento de la generación marcha a la par del gasto del consumo final privado $y$ el PIB nacional. Puesto en términos sencillos, esta relación que se ha observado también en otras regiones del mundo, se traduce en que a mayores niveles de consumo se produce un mayor volumen de residuos.

En el caso mexicano, entre 2003 y 2015, el producto interno bruto (PIB) y la generación de residuos crecieron prácticamente a la misma tasa (alrededor de $2.77 \%$ anual). 
Pruebas Iniciales para Construir Tarjetas Electrónicas Biodegradables.

Esta misma relación puede encontrarse a nivel de entidad federativa: aquellas que contribuyeron mayormente al PIB nacional también lo hicieron al volumen nacional de RSU.

No obstante, en algunos casos la contribución a la producción total nacional de RSU de algunas entidades se desvía notablemente de la tendencia general, como en el caso del estado de México (que produce más residuos respecto a la tendencia) o Campeche (que produce menos de lo esperado).

Esto se explica básicamente por la alta contribución al PIB del estado de México debida a su importan- te actividad industrial y a su alta población; mientras que, en el caso de Campeche, su comportamiento se debe a su intensa actividad petrolera y a su baja población. Si se clasifica a las entidades federativas por el volumen de RSU producidos, cinco concentraron el $45.7 \%$ en 2012: el estado de México (6.7 miIlones de toneladas; $16.1 \%$ del total nacional), el Distrito Federal (4.9 millones de toneladas; $11.8 \%$ ), Jalisco (3.1 millones de toneladas; $7.2 \%$ ), Veracruz (2.3 millones de toneladas; $5.5 \%)$ y Nuevo León (2.2 millones de toneladas; $5.1 \%$ ); mientras que las que registraron los menores volúmenes fueron Nayarit (347 mil toneladas; $0.82 \%$ ), Tlaxcala (339 mil toneladas; $0.81 \%)$, Campeche (272 mil toneladas; $0.65 \%)$,

\section{Figura 1. Entidates Federativas por volúmen de RSU producidos.}

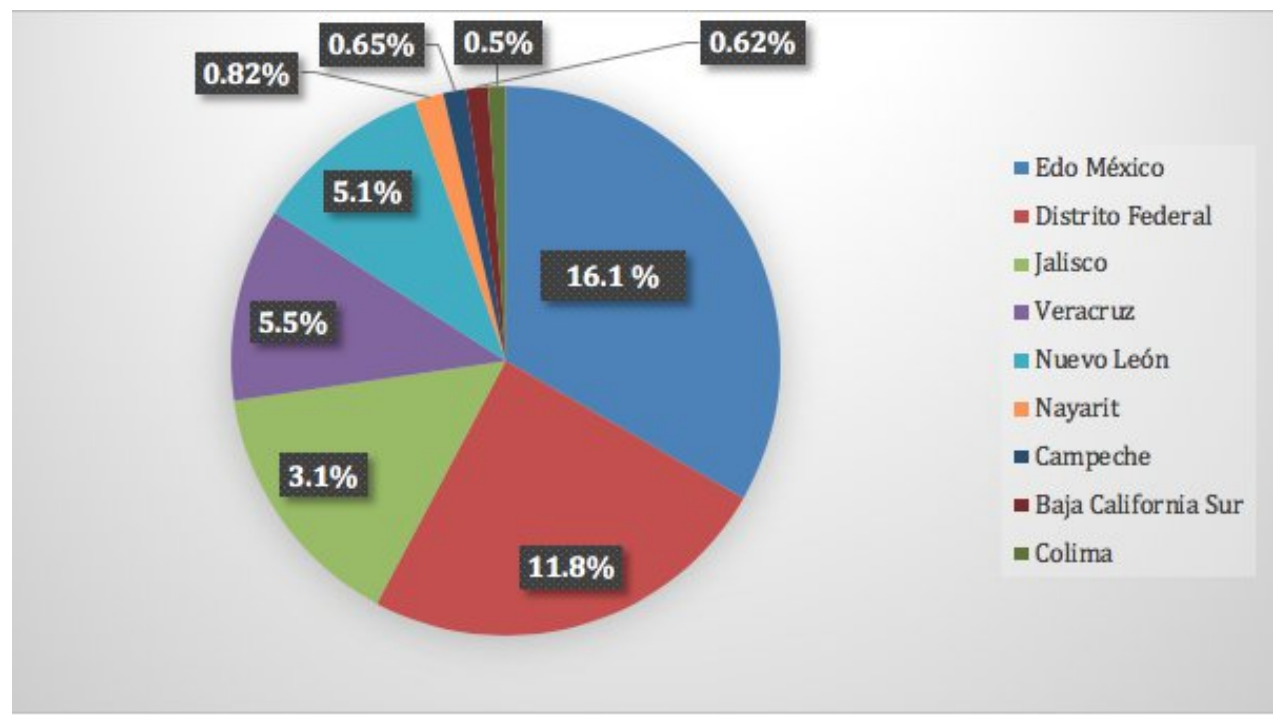


Baja California Sur (259 mil toneladas; $0.62 \%)$ y Colima (228 mil toneladas; $0.5 \%)$ (Kiss Köfalusi \& Encarnación Aguilar, 2006)(SEMARNAT, 2015) (Figura 1)

\section{Metodología}

Dentro de las instalaciones de desarrollo e innovación pertenecientes a la empresa ESTECS S.A de C.V, se realizó un análisis entre los procesos de construcción de tarjetas electrónicas implementando sustratos comunes y dos sustratos elaborados a partir de materiales biodegradables: Tetrapack ${ }^{\circledR}$ y material biobasado.

Los factores importantes a considerarse al seleccionar un material alterno para uso en tarjetas electrónicas son el grado de absorción de humedad, resistencia de aislamiento y constante dieléctrica.

Como primer sustrato alternativo se plantea el polietileno de baja densidad, elaborado a partir de bolsas de plástico reutilizadas y fibras de bagazo de caña (azúcar), que tiene propiedades similares a los anteriormente mencionadas, pero siendo semi-biodegradable y totalmente reciclable.
El segundo sustrato alternativo es el TetraPack $\AA$, un envase multicapas que se compone de tres materias primas: cartón $(70 \%)$, aluminio $(5 \%)$ y polietileno $(20 \%)$; este material se considera flexible por su alto porcentaje de cartón y presenta un tiempo de vida útil de 30 años(Tetrapak.com, 2016).

En la etapa de pruebas, se utilizó una impresora de circuitos para aplicar tinta conductiva sobre los tres diferentes sustratos (Figura 2).

Figura 2. Impresora de circuitos.

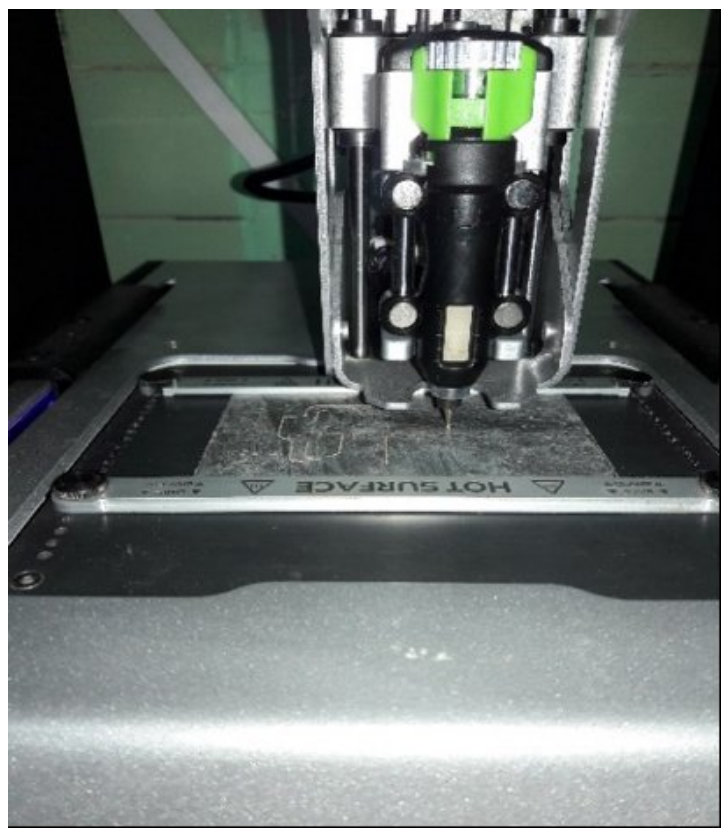

Fuente: Área de investigación, desarrollo e innovación, ESTECS S.A de C.V, 2018. 
Pruebas Iniciales para Construir Tarjetas Electrónicas Biodegradables.

Durante el proceso de impresión, se observó que en el material biobasado se presentaba una textura superficial rugosa, lo que provocó que las impresiones se realizarán en repetidas ocasiones para el mismo sustrato hasta obtener un circuito sin fallas (Figura 3 ).

\section{Figura 3. Impresión sobre sustrato biobasado.}

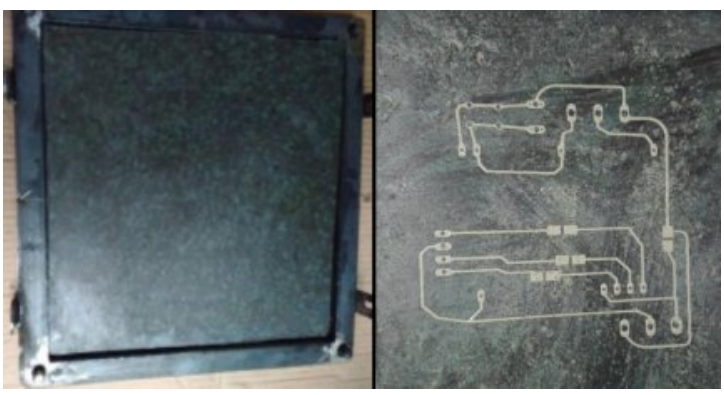

Fuente: Área de investigación, desarrollo e innovación, ESTECS S.A de C.V, 2018.

A diferencia del sustrato convencional en el sustrato con Tetrapack ${ }^{\circledR}$ donde la impresión de circuitos no presentó mayores problemas (figura 4).

Para el proceso de ensamble de los componentes electrónicos sobre las tres tarjetas de circuito impreso, se tiene que, en la tarjeta convencional, el proceso de ensamble fue más rápido con respecto a los dos sustratos alternativos.
Sin embargo, para la tarjeta con material biobasado nuevamente la textura no permitió un ensamble rápido, realizándose más intentos, sin obtener una tarjeta de calidad óptima para las etapas posteriores de operación.

Con respecto a la tarjeta en Tetrapack ${ }^{\circledR}$, se obtuvo un mejor circuito, y al ser un material de composición flexible, generó que, al colocar los elementos electrónicos con cautín, se presentarán deformaciones y quemaduras leves sobre su superficie, provocando problemas iniciales en el ensamble

\section{Figura 4. Impresión sobre sustrato Tretapack.}

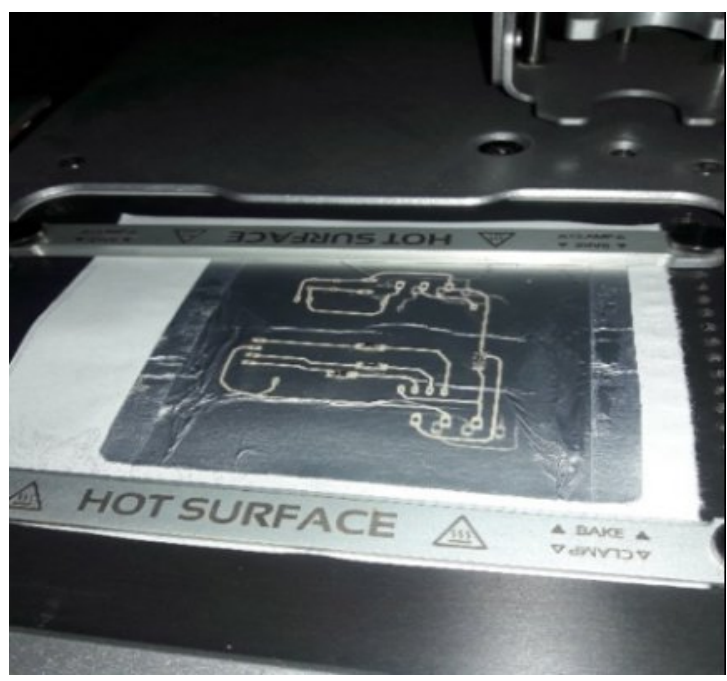

Fuente: Área de investigación, desarrollo e innovación, ESTECS S.A de C.V, 2018. 
total del circuito, y logrando un buen resultado al final del experimento.

Por ello, es preciso mencionar que los dos sustratos biodegradables propuestos no pueden ser sometidos a temperaturas mayores a $120^{\circ} \mathrm{C}$, debido a su poca tolerancia al calor, llegando a presentar deformaciones en las tarjetas y fracturas en las pistas.

Una vez ensambladas las tarjetas de circuitos sobre los tres sustratos, se instalaron una por una sobre un Sistema Eólico para probar su desempeño, realizando en éste las pruebas piloto de operación. En dichas pruebas, se observó que, de las tres tarjetas, dos de ellas (Tarjeta convencional y Tarjeta TetraPack $\left.{ }^{\circledR}\right)$ funcionaron sin mayores problemas mientras que, la tarjeta sobre material biobasado presentó deficiencias en la conductividad del circuito impreso y por ello no operó correctamente.

\section{RESUlTAdos y CONCLUSIO-} NES

En conclusión, se tiene que el sustrato generado de componentes termoestables (tarjeta electrónica común) es una buena opción si se requiere fabricar un circuito de larga duración pero sin ser una opción amigable para el ambiente, a diferencia del sustrato biobasado que está elaborado a partir de material reciclado y con alta posibilidad de reciclar el sustrato para usos posteriores, ayudando a incrementar el reciclaje de bolsas plásticas evitando la acumulación de las mismas en los rellenos sanitarios. Sin embargo, se sugiere mejorar la textura superficial mediante un proceso de pulido sobre el sustrato antes de iniciar la impresión de un circuito.

Por otro lado, se observa que la tendencia global se dirige a crear sistemas electrónicos de desecho rápido, es decir, que los tiempos de vida útil son cada vez más cortos. Con respecto a esto, el sustrato elaborado a partir de TetraPack $®$ tiende a ser una opción más viable 
Pruebas Iniciales para Construir Tarjetas Electrónicas Biodegradables.

para su uso como sustrato biodegradable en la elaboración de tarjetas electrónicas integradas en sistemas electrónicos de corta duración, recordando que este sustrato tarda 30 años aproximadamente en degradarse.

Considerando las características generales de este sustrato, es importante destacar que por el momento solo puede ser implementado en las etapas de control y operación digital, cuidando su uso en las etapas de potencia de los circuitos. No obstante, se pretende llevar estudios posteriores donde se genere un recubrimiento para este sustrato que permita alcanzar su uso óptimo y/o encontrar otro material para sustrato con bajo impacto al ambiente y de ser posible, totalmente biodegradable.

\section{AgRADECIMIENTOS}

La empresa ESTECS S.A de C.V agradece al Consejo de Ciencia y Tecnología (CONACYT) por su valioso apoyo para la ejecución de este proyecto de investigación.

\section{REFERENCIAS}

Allen, A. (2001). Containment landfills: the myth of sustainability. Engineering Geology, 60(1-4), 3-19.

https://doi.org/10.1016/S00137952(00)00084-3

Belloch, C. (2000). Recursos Tecnológicos (Tic). Unidad de Tecnología Educativa, 1-8. Retrieved from

https://www.uv.es/bellochc/logopedia/NRTLogo1.pdf

Corral Torres, S., Barrientos Becerra, B., Hernández Berriel, M. del C., Gómez Beltrán, G., \& Macedo Miranda, M. G. (2011). Afectación ambiental del tiradero a cielo abierto de Almoloya del Río, Estado de México. In S. Benitez, Ojeda, S. E. Cruz Sotelo, P. A. Taboada González, \& Q. Aguilar Virgen (Eds.), Hacia la sustentabilidad: Los residuos sólidos como fuente de energía y materia prima (pp. 253-257). Universidad Autónoma de Baja California.

Gupta, K. M., Gupta, N., \& Tiwari, A. (2015). Advanced Electrical and Electronics Materials: Processes and Applications. (I. Scrivener Publishing LLC y John Wiley \& Sons, Ed.). Wiley.

Kiss Köfalusi, G., \& Encarnación Aguilar, G. (2006). Los productos y los impactos de la descomposición de residuos sólidos urbanos en los sitios de disposición final. Gaceta Ecológica, (79), 39-51.

Martínez Rivera, C. (2008). A China la basura electrónica. Retrieved December 29, 2018, from http://www.adendi.com/archi-

vo.asp? num $=57535 \&$ year $=2008 \&$ month $=7 \&$ keyword $=$ 
Mexicana, D., Carrillo, C., Pr, M. S. D., Toluca, P., \& Licenciatura, P. (2009). Materiales de resinas compuestas y su polimerización. Revista ADM Organo Oficial de La Asociacion Dental Mexicana., LXV(4), 10-17.

Molina, M. J., \& Rowland, F. S. (1974). Stratospheric sink for chlorofluoromethanes: chlorine atom-catalysed destruction ozone. Nature, 249(5460), 810-812.

SEMARNAT. (2015). Informe de la Situación del Medio Ambiente en México. In Informe de la Situación del Medio Ambiente en México. Compendio de Estadísticas Ambientales. (Primera Ed). Ciudad de México. Retrieved from https://apps1.semarnat.gob.mx:445/dgei a/informe15/tema/pdf/Cap7_Residuos.pd $f$

Tetrapak.com. (2016). Tetra Brick envases. Retrieved December 29, 2018, from https://www.tetrapak.com/ 